DOI: $10.17957 / \mathrm{IJAB} / 15.1732$

http://www.fspublishers.org

\title{
Plasmodiophora brassicae in Yunnan and its Resistant Sources in Chinese Cabbage
}

\author{
Xueyu Han ${ }^{1 \dagger}$, Junlong Yin ${ }^{1,2 \dagger}$, Ikram Ullah ${ }^{1}$, Enzhu Luo ${ }^{1}$ and Yanling Yue ${ }^{1^{*}}$ \\ ${ }^{1}$ College of Landscape and Horticulture, Yunnan Agricultural University, Kunming 650201, China \\ ${ }^{2}$ Chinese Academy of Tropical Agricultural Sciences, Proving Ground, Danzhou 571737, China \\ *For correspondence: yanling-yue@126.com; 2217714953@qq.com \\ ${ }^{\dagger}$ Contributed equally to this work and are co-first authors \\ Received 01 August 2020; Accepted 02 January 2021; Published 25 March 2021
}

\begin{abstract}
The Williams differential system was employed for pathotype identification of 34 Plasmodiophora brassicae root samples collected from Yunnan Province and pathotypes 1, 2, 4, 10 and 14 were detected. Pathotype 4 was dominant with $70.59 \%$ of all the samples in Yunnan. The distribution of the P. brassicae pathotypes was mapped. Resistance to $P$. brassicae (clubroot disease) was investigated in 22 Chinese cabbage cultivars and it was found that the cultivar Shangpin had multiple resistances and was immune while Shangpin CR527 and Shangpin CR523 were resistant to P. brassicae. These cultivars can be used by farmers as sources of resistance to $P$. brassicae, to aid them in reducing disease in their crops. Seven known clubroot-resistant genes were detected in the 22 Chinese cabbage cultivars. $C R a$ and $C R b$ were found to be the most resistant to $P$. brassicae pathotype 4. Beisheng CR12 was resistant to pathotypes 1, 4, 10 and 14, but did not carry any known resistance genes, which indicated that unknown resistant genes were present. This study will lay the foundation for the control of clubroot disease and promote disease-resistant breeding of Chinese cabbage. (C) 2021 Friends Science Publishers
\end{abstract}

Keywords: Clubroot; Differential hosts; Plasmodiophora brassicae; Resistance identification

\section{Introduction}

Clubroot, caused by Plasmodiophora brassicae $(P$. brassicae), is an obligate parasitic soil-borne disease of plants causing 25-60\% of yield losses in crucifer crops annually (Faggian et al. 1999; Howard et al. 2010; Suo et al. 2015). P. brassicae infects the roots, causing swelling or formation of galls on the roots. The distorted root reduces water and nutrient uptake and leaf heads fail to develop (Faggian et al. 1999). Clubroot was first reported in 1737 on the west coast of the Mediterranean Sea and southern Europe (Karling 1942). In Asia, it was first reported in Taiwan, China (1936) and then spread to Japan and Korea. At present, there are reports of clubroot occurrence in the Northeast, Southwest, Shandong and the upper and middle reaches of the Yangtze River in China (Suo et al. 2015). The resting spores of $P$. brassicae can survive for more than a decade within the soil (Karling 1942), even if the cruciferous crops were no longer cultivated. Once a suitable host is available for infection, the resting spores activate and invade the roots, and additional resting spores are released after root decomposition, thus threatening the sustainable production of cruciferous vegetables (Fei et al. 2015). Some measures have been taken to control this pathogen in infested fields. Crop rotation is commonly used to prevent the spread of clubroot disease (Howard et al. 2010), however, this method is not a practical way to eradicate spores (Peng et al. 2015). Soil amendments with lime have also been considered as an effective management strategy to reduce clubroot infestation, by increasing soil $\mathrm{pH}(\mathrm{Lv}$ et al. 2018). However, the repetitive use of lime destroys the soil structure, ultimately affecting sustainable production (Webster and Dixon 1991). Fluazinam, a synthetic fungicide, has been shown to control P. brassicae (Howard et al. 2010; Yuan et al. 2016), however, improper use of the soil and plant residues greatly affects human health and environmental safety (Lee et al. 2012). Endophytic actinomycetes isolated from the roots of Chinese cabbage can effectively control clubroot (Lee et al. 2008), but the activity against $P$. brassicae is susceptible to soil environmental conditions, and their field efficacy is inconsistent and often ineffective (Saravanan et al. 2003; Mcgrann et al. 2017). Wang et al. (2016) found that the Streptomyces albospinus CT205, proved to be effective in preventing clubroot disease, although environmental and climatic factors severely affect its performance. Biocontrol agents are expensive and, if not applied properly, can lead to an increased production cost. Therefore, the application of 
resistant varieties is considered to be one of the most effective and environmentally friendly approaches for treating clubroot disease.

Clubroot has been present in Yunnan for approximately 30 years. It first appeared in the Chenggong area of Kunming and has been identified in Chuxiong, Yuxi, Qujing, Dali, and other areas. Yunnan is the main vegetable producing area in China, and Chinese cabbage is an important export vegetable of Yunnan Province, cultivated in an area of approximately $100000 \mathrm{hm}^{2}$ with a yield of 4.06 million. The soil contaminated by $P$. brassicae in the main production regions accounts for approximately $20 \%$ of the total area and the loss of crops is generally $20-40 \%$, but can be up to $100 \%$. Several resistant Chinese cabbage varieties, such as CR Huimin, Kanggen 51 and Tiejia No.1, have been developed and introduced (Tan and Yue 2013; Yin et al. 2018), although these studies are mostly directed at a single $P$. brassicae pathotype and a single sampling site, lacking comprehensive evaluation of pathotype differentiation, variety resistance and a suitable applied range in the province. In order to fully understand the distribution of clubroot in Yunnan Province, $P$. brassicae samples were collected from 34 different clubroot growing areas in 2017-2019 and their pathotypes and resistant cultivars of Chinese cabbage were identified, which would provide useful information for farmers to prevent and control clubroot.

\section{Materials and Methods}

\section{Plant pathogen materials}

A total of $34 P$. brassicae samples were collected from the main cruciferous vegetable growing areas in Yunnan Province. The samples, along with collection sites and hosts, are shown in Table 1. For pathotype identification of the 34 $P$. brassicae samples, the hosts proposed by Williams, including two cabbage cultivars Jersey Queen (JQ), Badger Shipper (BS) and two turnip cultivars Laurentian (LT), Wilhelmsburger (WB), were obtained from the Liaoning Academy of Agricultural Sciences, China. Twenty-one Chinese cabbage-resistant varieties and the susceptible control variety '83-1'were purchased from markets.

Preparation of the pathogen, plant inoculation and disease assessment

Resting spores were extracted from samples of $P$. brassicae using the method described by Zheng et al. (2019). The peat and resting spores were mixed evenly, with $1 \times 10^{7}$ spores per gram of pathogen soil. Peat, perlite, and vermiculite with a volume ratio of 2:1:1 were mixed and packed in seedling plugs with 72 holes, and 1.0 grams of pathogen soil was added to each hole. Germinated seeds of Williams' identified hosts, and 22 Chinese cabbage varieties were sowed on pathogen soil, one seed per hole, and one seedling plug per variety. They were fertilised with Hoagland nutrient solution in the greenhouse (average night temperature $18^{\circ} \mathrm{C}$ and average day temperature $25^{\circ} \mathrm{C}$ ). After 40 days of inoculation, pathotypes of $34 P$. brassicae were identified according to the resistant and susceptible responses by Williams' hosts (Williams 1996). The severity of the club development on each plant was rated using a scale of $0,1,3,5,7$ and 9, according to The Grading Standard for Clubroot Resistance of Brassicaceae Crop Seedling Period, where $0=$ no clubbing, $1=$ one or a few small clubs on the lateral roots, $3=$ clubs on the main roots with diameters less than 2 times that of the stem base, $5=$ clubs on the main roots with diameters 2-3 times that of the stem base, $7=$ clubs on the main roots, with diameters 3-4 times that of the stem base, and $9=$ clubs on the main roots with diameters more than four times that of the stem base, or the swollen root is blackening. The index of disease (ID) was calculated using the following formula:

$$
\text { ID }(\%)=\sum(n \times 0+n \times 1+n \times 3+n \times 5+n \times 7+n \times 9) /(N \times 9) \times 100
$$

where $\mathrm{n}$ is the number of plants in each class, $\mathrm{N}$ is the total number of plants, and $0,1,3,5,7$ and 9 are the symptom severity classes.

\section{DNA extraction and primer design}

The Plant DNA Extraction Kit (http://www.bioteke.com/) was used to extract DNA from the leaves of 22 Chinese cabbage varieties and 4 Williams' hosts, according to the manufacturer's instructions. Primers for disease-resistant genes were designed based on published molecular marker sequences of CR loci: Crrl (Suwabe et al. 2003), Crr2 (Suwabe et al. 2003), Crr3 (Hirai et al. 2004), CRa (Ueno et al. 2012), CRb (Hatakeyama et al. 2013), CRc (Ueno et al. 2012) and $C R k$ (Ueno et al. 2012). The list of primers used is listed in Table 2.

\section{PCR reaction procedure and gel electrophoresis}

Each locus was amplified with 2 ng of template DNA in a $25 \mu \mathrm{L}$ reaction volume containing $1 \mu \mathrm{L}$ of each primer and $12.5 \mu \mathrm{L}$ of $2 \times$ PCR master mix (Takara, Japan). Thermal cycling conditions comprised denaturation at $94^{\circ} \mathrm{C}$ for 3 min, followed by 35 cycles of denaturation at $94^{\circ} \mathrm{C}$ for $30 \mathrm{~s}$, annealing temperature of each primer at $61^{\circ} \mathrm{C}, 55.5^{\circ} \mathrm{C}$, $55.5^{\circ} \mathrm{C}, 54.5^{\circ} \mathrm{C}, 57^{\circ} \mathrm{C}, 52.2^{\circ} \mathrm{C}, 49^{\circ} \mathrm{C}, 47^{\circ} \mathrm{C}$ for $30 \mathrm{~s}$ and extension at $72^{\circ} \mathrm{C}$ for $30 \mathrm{~s}$ followed by extension at $72^{\circ} \mathrm{C}$ for 5 min in a BioTeke GeneAmp PCR system. Amplified PCR products were separated by electrophoresis in a $1.5 \%$ agarose gel in Tris-acetate-EDTA buffer, stained with EB (ethidium bromide) and photographed.

\section{Results}

\section{Identification of pathotypes}

The total collection of 34 P. brassicae isolates was assessed. The susceptible or resistant responses of Williams' hosts to $P$. brassicae are shown in Table 3 . The $34 P$. brassicae 
Table 1: Sampling codes, site and hosts of 34 Plasmodiophora brassicae samples

\begin{tabular}{|c|c|c|c|c|c|c|c|c|c|c|c|}
\hline Code & Site & Hosts & Code & Site & Hosts & Code & Site & Hosts & Code & Site & Hosts \\
\hline$\overline{\mathrm{P} 1}$ & Songming & Chinese cabbage & $\mathrm{P} 2$ & Songming & Chinese cabbage & P3 & Yingjiang & Chinese cabbage & $\mathrm{P} 4$ & Yuxi & Chinese cabbage \\
\hline P5 & Panlong & Chinese cabbage & P6 & Lufeng & Chinese cabbage & P7 & Yaoan & Chinese cabbage & P8 & Nanhua & Chinese cabbage \\
\hline P9 & Chenggong & Chinese cabbage & $\mathrm{P} 10$ & Yingjiang & Chinese cabbage & P11 & Jianshui & Chinese cabbage & $\mathrm{P} 12$ & Jianshui & Chinese cabbage \\
\hline P13 & Ludian & Chinese cabbage & $\mathrm{P} 14$ & Yiliang & Brassica oleracea & P15 & Yiliang & Chinese cabbage & P16 & Luliang & Chinese cabbage \\
\hline P17 & Luliang & Chinese cabbage & P18 & Qujing & Chinese cabbage & P19 & Dali & Chinese cabbage & $\mathrm{P} 20$ & Luquan & Chinese cabbage \\
\hline $\mathrm{P} 21$ & Ludian & Chinese cabbage & $\mathrm{P} 22$ & Ludian & Chinese cabbage & $\mathrm{P} 23$ & Xundian & Cauliflower & $\mathrm{P} 24$ & Luliang & Brassica oleracea \\
\hline $\mathrm{P} 25$ & Anning & Cauliflower & P26 & Wuding & Chinese cabbage & $\mathrm{P} 27$ & Shilin & Chinese cabbage & P28 & Jinning & Chinese cabbage \\
\hline $\mathrm{P} 29$ & Fumin & Chinese cabbage & P30 & Xishan & Chinese cabbage & P31 & Weishan & Chinese cabbage & P32 & Tonghai & Chinese cabbage \\
\hline P33 & Xuanwei & Chinese cabbage & P34 & Qiubei & Chinese cabbage & & & & & & \\
\hline
\end{tabular}

Table 2: Primers used for detection of clubroot resistant genes

\begin{tabular}{|c|c|c|c|c|}
\hline Locus & Primer name & Primer sequences & Product size/bp & References \\
\hline \multirow[t]{2}{*}{ Crrl } & BRMS-088-FW & TATCGGTACTGATTCGCTCTTCAAC & R263/S233 & (Suwabe et al. 2003). \\
\hline & BRM-088-RV & ATCGGTTGTTATTTGAGAGCAGATT & & \\
\hline \multirow[t]{2}{*}{$\mathrm{Cr} 2$} & BRMS-096-FW & AGTCGAGATCTCGTTCGTGTCTCCC & R220/S189 & (Suwabe et al. 2003). \\
\hline & BRMS-096-RV & TGAAGAAGGATTGAAGCTGTTGTTG & & \\
\hline \multirow[t]{2}{*}{ Crr3 } & OPC11-2F & GTAACTTGGTACAGAACAGCATAG & R1300/S1000 & (Hirai et al. 2004). \\
\hline & OPC11-2R & ACTTGTCTAATGAATGATCATGG & & \\
\hline \multirow[t]{3}{*}{$C R a$} & SC2930-T-FW & TAGACCTTTTTTTTGTCTTTTTTTTTAC & $\mathrm{R} 800$ & (Ueno et al. 2012). \\
\hline & SC2930-Q-FW & CAGACTAGACTTTTTGTCATTTAGA & S800 & \\
\hline & SC2930-RV & CTAAGGCCATAGAAATCAGGTC & & \\
\hline \multirow{2}{*}{$C R b$} & KBrH129J18R-FW & AGAGCAGAGTGAAACCAGAACT & $\mathrm{R} 254 / \mathrm{S} 194$ & (Hatakeyama et al. 2013). \\
\hline & KBrH129J18R-RV & GTTTCAGTTCAGTCAGGTTTTTGCAG & & \\
\hline \multirow[t]{3}{*}{$C R c$} & B50-C9-FW & GATTCAATGCATTTCTCTCGAT & R800 & (Ueno et al. 2012). \\
\hline & B50-6R-FW & AATGC ATTTTCGCTC AACC & S800 & \\
\hline & B50-RV & CGTATT ATATC TCTTT CTCCA TCCC & & \\
\hline \multirow[t]{3}{*}{$C R k$} & HC688-4-FW & TCTCTG TATTGCGTTGACTG & R1000 & (Ueno et al. 2012). \\
\hline & HC688-6-RV & ATATGTTGAAGCCTATGTCT & $\mathrm{S} 1000$ & \\
\hline & HC688-7-RV & AAATATATGTGAAGTCTTATG ATC & & \\
\hline
\end{tabular}

Table 3: Resistant and susceptible response (severity based on a disease index, DI) of Williams' differential hosts to collections of Plasmodiophora brassicae from Yunnan Province, China

\begin{tabular}{|c|c|c|c|c|c|c|c|c|c|c|c|c|c|c|c|c|c|c|c|}
\hline \multirow[t]{2}{*}{ Code } & \multicolumn{8}{|c|}{ Williams hosts and disease index } & \multirow[t]{2}{*}{ Pathotypes } & \multirow[t]{2}{*}{ Code } & \multicolumn{8}{|c|}{ Williams hosts and disease index } & \multirow[t]{2}{*}{ Pathotypes } \\
\hline & JQ & DI & BS & DI & LT & DI & WB & DI & & & JQ & DI & BS & DI & LT & DI & WB & DI & \\
\hline P1 & + & 44 & + & 16 & + & 50 & + & 11 & 4 & P18 & + & 13 & + & 26 & + & 7 & - & 0 & 2 \\
\hline $\mathrm{P} 2$ & + & 21 & + & 33 & + & 28 & + & 30 & 4 & P19 & + & 36 & + & 15 & + & 20 & + & 11 & 4 \\
\hline P4 & + & 26 & + & 61 & + & 11 & + & 35 & 4 & $\mathrm{P} 21$ & + & 12 & + & 11 & + & 16 & + & 11 & 4 \\
\hline P5 & + & 56 & + & 74 & + & 59 & + & 42 & 4 & $\mathrm{P} 22$ & + & 32 & + & 17 & + & 41 & + & 39 & 4 \\
\hline P6 & + & 19 & + & 31 & + & 40 & - & 0 & 2 & $\mathrm{P} 23$ & + & 17 & + & 11 & - & 0 & + & 35 & 10 \\
\hline P7 & + & 26 & + & 44 & + & 47 & + & 22 & 4 & $\mathrm{P} 24$ & + & 23 & + & 21 & + & 22 & + & 42 & 4 \\
\hline P8 & + & 31 & + & 34 & + & 46 & + & 19 & 4 & $\mathrm{P} 25$ & + & 20 & + & 20 & + & 13 & + & 13 & 4 \\
\hline P9 & + & 31 & + & 11 & + & 19 & + & 35 & 4 & P26 & + & 44 & + & 24 & + & 48 & + & 42 & 4 \\
\hline P10 & + & 40 & + & 35 & + & 27 & + & 17 & 4 & $\mathrm{P} 27$ & + & 40 & + & 11 & - & 0 & + & 11 & 10 \\
\hline P11 & + & 26 & + & 32 & + & 26 & + & 42 & 4 & P 28 & + & 43 & + & 17 & + & 11 & + & 18 & 4 \\
\hline P12 & + & 20 & + & 22 & + & 12 & + & 19 & 4 & P29 & + & 28 & + & 46 & + & 34 & + & 38 & 4 \\
\hline P13 & + & 7 & + & 11 & - & 0 & + & 35 & 10 & P30 & + & 20 & + & 20 & + & 15 & + & 23 & 4 \\
\hline P14 & + & 21 & - & 0 & + & 21 & + & 21 & 1 & P31 & + & 38 & + & 19 & - & 0 & + & 27 & 10 \\
\hline P15 & + & 15 & + & 15 & + & 22 & + & 24 & 4 & P32 & + & 30 & + & 50 & + & 13 & + & 18 & 4 \\
\hline
\end{tabular}

JQ, BS, LT, and WB are Williams hosts (Jersey Queen, Badger Shipper, Laurentian, Wilhelmsburger). P1-P34 are the 34 P. brassicae samples. "Pathotypes": the identified result of Pathotypes

could be classified into five pathotypes: pathotypes 1, 2, 4, 10 and 14 . Among them, 24 samples were pathotype 4, accounting for $70.59 \%$ of the total samples (Table 3 ). The results indicated that pathotype 4 was the predominant pathotype in Yunnan Province. All the Williams' hosts (JQ, $\mathrm{BS}$, LT and WS) were susceptible to $P$. brassicae of pathotype 4 , which confirmed that this variety had the strongest pathogenicity of those studied.
The disease index of hosts JQ, BS, LT and WS differed after inoculation with pathotype 4 . The disease index of JQ was 12 in $\mathrm{P} 21$ but reached 85 in $\mathrm{P} 3$, whereas the disease index of BS was 11 in P9 and P21, but 74 in P5. Similarly, the results for LT ranged from 11 to 59 and the disease index of WS ranged from 11 to 54 for pathotype 4 . This shows pathogenic diversity among $P$. brassicae of pathotype 4 in Yunnan Province. The disease index of hosts 


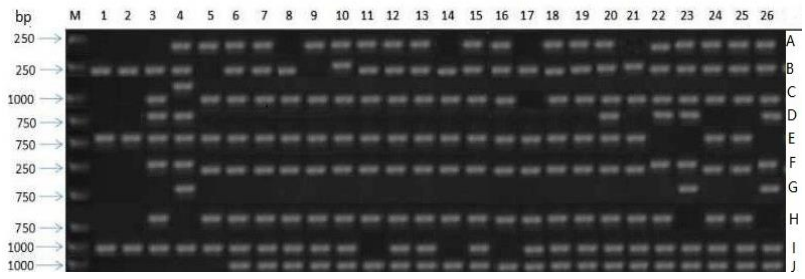

Fig. 1: Amplification results of different resistant genes Note: M: Marker DL2000; 1-4: Williams Identification Hosts Badger Shipper, Jersey Queen, Wilhelmsburger, Laurentian; 5: CK cultivars 83-1; 6-26: cultivars Chunqiushengen, CR65, Tianci, Jinfu baby cabbage, Guizu, Degaorongyao, Kanggen 911, Taineng CR119, Beisheng CR12, Chinese cabbage King, CR mogen, Xinkanggen, CR gaokangwang, Kangbingwang CR117, Guoshen CR167, Shenggen No.1, Shangpin, Shangpin CR527, Degao CR117, Tiejia No.1, Shangpin CR523. A, B, C, (D, E), F, (G, H), (I, J): amplification results of Crrl (BRMS-088), Crr2 (BRMS-096), Crr3 (OPC11-2), CRa (SC2930-T, SC2930-Q), CRb (KBrH129J18R), CRc (B50-C9, B50-6R), CRk (HC688-6, HC688-7) respectively. The product size of resistant and susceptible is shown in Table 3

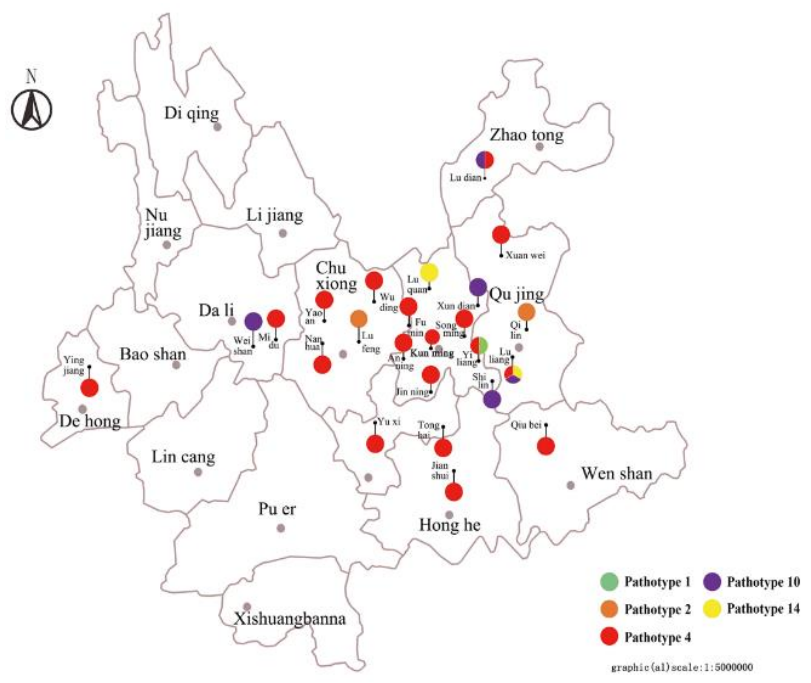

Fig. 2: Distribution of pathotypes of Plasmodiophora brassicae in Yunnan

was higher in P3 and P5 than in others, indicating that the pathogenicity of $\mathrm{P} 3$ and $\mathrm{P} 5$ was stronger than that of other P. brassicae of pathotype 4 .

\section{Diseases reaction of Chinese cabbage cultivars}

We assessed the disease responses of 22 Chinese cabbage cultivars to $34 P$. brassicae isolates (Table 4). The control variety (83-1) showed the typical clubroot symptoms with all $P$. brassicae isolates, which indicates that the method of inoculation was suitable. The other 21 Chinese cabbage varieties exhibited different resistance responses to the $34 P$. brassicae isolates. Shangpin was immune to each of the 34 collections, whereas the disease index of Shangpin CR527 and Shangpin CR523 were less than 10, indicating strong resistance. From the resistance expression of cultivars to pathotype 4, there were only three cultivars namely Shangpin, Shangpin CR527 and Shangpin CR523 that were immune to P5, less than the other varieties included in pathotype 4, which shows that P5 was a stronger version of $P$. brassicae. There were few sources which showed resistance to $\mathrm{P} 5$.

As shown in Table 4, CR8, CR9, CR10, CR17, CR18 and CR21 expressed immunity to P14 (pathotype 1). The disease indices of CR2, CR3, CR5, CR13, CR14 and CR16 were lower than 10 , indicating that they were resistant to P14. CR17, CR18 and CR21 exhibited immunity, while CR1 and CR10 were resistant to P6 and P18 (pathotype 2). CR9, CR17, CR18 and CR21 were immune, and CR12 and CR16 were resistant to P13, P17, P23, P27 and P31 (pathotype 10). CR9, CR13, CR14, CR16, CR17, CR18, CR19 and CR21 showed immunity, and CR1 and CR8 were resistant to P16 and P20 (pathotype 14).

\section{Molecular markers for resistance}

To investigate the relationship between the clubrootresistant varieties and known CR resistance loci, PCR amplification of the seven known molecular markers was applied for genotyping Williams' hosts and the 22 cultivars.

The results (Fig. 1, Table 5) indicated that LT contained the most disease-resistant genes: $\mathrm{Crr} 3, \mathrm{CR} a, \mathrm{CR}$, $C R c$ and $C R k$. WB contained three disease-resistant genes: $C R a, C R b$ and $C R k$. Only the resistant gene $C R k$ was detected in the BS and JQ. Among the 21 tested varieties, CR18 and CR21 contained four disease-resistant genes, heterozygous $C R a$ and $C R k$, homozygous $C R b$ and $C R c$, which accounted for $9.52 \%$ of the total tested varieties. CR17 was checked for three disease-resistant genes, homozygous $C R a, C R b$ and heterozygous $C R k$, which accounted for $4.76 \%$. CR5 was examined for two resistant genes, homozygous $\mathrm{Cr} 2$ and heterozygous $C R k$, accounting for $4.76 \%$. CR15 contained two resistant genes, heterozygous $C R a$ and $C R k$, which accounted for $4.76 \%$, and CR7, CR9 and CR11 did not test for any diseaseresistant genes, accounting for $14.29 \%$ of the total tested cultivars. The other cultivars contained only one heterozygous $C R k$ gene (61.9\%).

\section{Discussion}

There are few reports related to the study of pathotype differentiation of $P$. brassicae in China, and only a few pathotypes have been detected, such as pathotype 4 in areas of Shandong and Jilin Province and pathotypes 2, 4 and 11 in the Liaoning area (Shen et al. 2009). Pathotypes 7 and 11 have been reported in the Sichuan area (Chen et al. 2016), and pathotypes 1, 4, 9 and 13 in the Hunan area (Peng et al. 2013). The clubroot in the Shandong, Jilin, Liaoning, Sichuan, and Hunan areas could be explained by the cultivation of resistant varieties. In this study, 5 pathotypes, pathotype 1, 2, 4, 10 and 14, were investigated in Yunnan Province and only Shangpin was determined as immune to all pathotypes, indicating that $P$. brassicae in Yunnan is more complex and pathogenic than in other areas in China. 
Table 4: Resistant and susceptible response (severity based on a disease index, DI) of 22 differential varieties to collections of Plasmodiophora brassicae from Yunnan Province, China

\begin{tabular}{|c|c|c|c|c|c|c|c|c|c|c|c|c|c|c|c|c|c|c|c|c|c|c|c|}
\hline Varie & & 83-1 & CR1 & CR2 & CR3 & CR4 & CR5 & CR6 & CR7 & CR8 & CR9 & CR10 & CR11 & CR12 & CR13 & CR14 & CR15 & CR16 & CR17 & CR18 & CR19 & CR20 & CR21 \\
\hline \multirow[t]{2}{*}{ P1 } & DI & 61 & 0 & 4 & 0 & 43 & 16 & 10 & 0 & 0 & 0 & 0 & 3 & 0 & 0 & 0 & 17 & 2 & 0 & 0 & 0 & 12 & \\
\hline & $\mathrm{R}$ & $S$ & I & $\mathrm{R}$ & I & $S$ & $S$ & $S$ & I & I & I & I & $\mathrm{R}$ & I & I & I & $S$ & $\mathrm{R}$ & I & I & I & $\mathrm{S}$ & $\mathrm{R}$ \\
\hline \multirow[t]{2}{*}{ P2 } & DI & 57 & 31 & 4 & 5 & 70 & 5 & 15 & 11 & 0 & 0 & 0 & 52 & 0 & 2 & 0 & 57 & 11 & 0 & 0 & 2 & 23 & 5 \\
\hline & $\mathrm{R}$ & S & S & $\mathrm{R}$ & $\mathrm{R}$ & $\mathrm{S}$ & $\mathrm{R}$ & S & $\mathrm{S}$ & I & I & I & $\mathrm{S}$ & I & $\mathrm{R}$ & I & S & $\mathrm{S}$ & I & I & $\mathrm{R}$ & $\mathrm{S}$ & $\mathrm{R}$ \\
\hline \multirow[t]{2}{*}{ P3 } & DI & 85 & 19 & 57 & 9 & 43 & 33 & 41 & 0 & 0 & 0 & 1 & 43 & 0 & 19 & 5 & 49 & 32 & 0 & 0 & 13 & 0 & 0 \\
\hline & $\mathrm{R}$ & $S$ & $\mathrm{~S}$ & $\mathrm{~S}$ & $R$ & S & S & S & I & I & I & $\mathrm{R}$ & S & I & $S$ & $\mathrm{R}$ & $\mathrm{S}$ & $S$ & I & I & $\mathrm{S}$ & I & I \\
\hline P4 & DI & 72 & 25 & 42 & 52 & 58 & 15 & 35 & 17 & 0 & 0 & 0 & 30 & 11 & 7 & 10 & 56 & 17 & 0 & 0 & 38 & 14 & 0 \\
\hline & $\mathrm{R}$ & $\mathrm{S}$ & $\mathrm{S}$ & $\mathrm{S}$ & $\mathrm{S}$ & $\mathrm{S}$ & $\mathrm{S}$ & S & $\mathrm{S}$ & I & I & I & $\mathrm{S}$ & $S$ & $\mathrm{R}$ & $S$ & $\mathrm{~S}$ & $S$ & I & I & $\mathrm{S}$ & $\mathrm{S}$ & I \\
\hline P5 & DI & 86 & 26 & 41 & 31 & 37 & 79 & 19 & 43 & 31 & 31 & 33 & 50 & 30 & 31 & 33 & 48 & 50 & 0 & 0 & 46 & 54 & 0 \\
\hline & $\mathrm{R}$ & $\mathrm{S}$ & $\mathrm{S}$ & $S$ & $\mathrm{~S}$ & $\mathrm{~S}$ & S & S & $\mathrm{S}$ & S & $S$ & S & $\mathrm{S}$ & $\mathrm{S}$ & $\mathrm{S}$ & $\mathrm{S}$ & S & $\mathrm{S}$ & I & I & $S$ & $\mathrm{~S}$ & I \\
\hline P6 & DI & 65 & 3 & 20 & 11 & 65 & 14 & 28 & 32 & 38 & 48 & 0 & 11 & 17 & 10 & 27 & 80 & 27 & 0 & 0 & 19 & 25 & 0 \\
\hline & $\mathrm{R}$ & $\mathrm{S}$ & $\mathrm{R}$ & S & $\mathrm{S}$ & S & $\mathrm{S}$ & S & $\mathrm{S}$ & $\mathrm{S}$ & $\mathrm{S}$ & I & $\mathrm{S}$ & S & $\mathrm{S}$ & S & $\mathrm{S}$ & S & I & I & $\mathrm{S}$ & $\mathrm{S}$ & I \\
\hline P7 & DI & 80 & 33 & 78 & 19 & 62 & 56 & 19 & 39 & 20 & 4 & 0 & 10 & 0 & 1 & 27 & 48 & 46 & 0 & 0 & 11 & 15 & 0 \\
\hline & $\mathrm{R}$ & $\mathrm{S}$ & S & S & $\mathrm{S}$ & $\mathrm{S}$ & S & $S$ & S & $\mathrm{S}$ & $\mathrm{R}$ & I & $\mathrm{S}$ & I & $\mathrm{R}$ & $\mathrm{S}$ & $\mathrm{S}$ & $\mathrm{S}$ & I & I & $\mathrm{S}$ & $\mathrm{S}$ & I \\
\hline P8 & DI & 67 & 17 & 26 & 0 & 41 & 48 & 22 & 0 & 0 & 8 & 2 & 53 & 23 & 0 & 0 & 27 & 27 & 0 & 0 & 13 & 12 & 0 \\
\hline & $\mathrm{R}$ & $\mathrm{S}$ & $\mathrm{S}$ & $\mathrm{S}$ & I & $\mathrm{S}$ & $\mathrm{S}$ & $\mathrm{S}$ & I & I & $\mathrm{R}$ & $\mathrm{R}$ & $\mathrm{S}$ & $S$ & I & I & $S$ & $S$ & I & I & $\mathrm{S}$ & $\mathrm{S}$ & I \\
\hline P9 & DI & 56 & 11 & 26 & 17 & 59 & 33 & 19 & 56 & 19 & 16 & 20 & 4 & 0 & 0 & 36 & 11 & 23 & 0 & 0 & 35 & 28 & 2 \\
\hline & $\mathrm{R}$ & $\mathrm{S}$ & $\mathrm{S}$ & S & $\mathrm{S}$ & S & $\mathrm{S}$ & S & S & S & S & $S$ & $\mathrm{R}$ & I & I & S & $\mathrm{S}$ & S & I & I & S & S & $\mathrm{R}$ \\
\hline P10 & DI & 72 & 4 & 9 & 2 & 46 & 16 & 16 & 32 & 0 & 38 & 0 & 29 & 33 & 6 & 7 & 41 & 26 & 0 & 0 & 31 & 13 & 0 \\
\hline & $\mathrm{R}$ & $\mathrm{S}$ & $\mathrm{R}$ & $\mathrm{R}$ & $\mathrm{R}$ & $\mathrm{S}$ & $\mathrm{S}$ & $\mathrm{S}$ & $\mathrm{S}$ & I & $\mathrm{S}$ & I & S & $\mathrm{S}$ & $\mathrm{R}$ & $\mathrm{R}$ & $\mathrm{S}$ & $S$ & I & I & $\mathrm{S}$ & $\mathrm{S}$ & I \\
\hline P11 & DI & 65 & 1 & 19 & 0 & 47 & 28 & 23 & 42 & 17 & 22 & 1 & 15 & 19 & 0 & 0 & 70 & 7 & 0 & 0 & 7 & 7 & 0 \\
\hline & $\mathrm{R}$ & $S$ & $\mathrm{R}$ & $\mathrm{S}$ & I & $S$ & $\mathrm{~S}$ & S & $\mathrm{S}$ & $\mathrm{S}$ & $\mathrm{S}$ & $\mathrm{R}$ & S & $\mathrm{S}$ & I & I & $\mathrm{S}$ & $\mathrm{R}$ & I & I & $\mathrm{R}$ & $\mathrm{R}$ & I \\
\hline P12 & DI & 76 & 9 & 13 & 0 & 31 & 29 & 0 & 21 & 0 & 4 & 0 & 29 & 37 & 4 & 11 & 51 & 4 & 0 & 0 & 0 & 36 & 0 \\
\hline & $\mathrm{R}$ & $\mathrm{S}$ & $\mathrm{R}$ & S & I & S & $\mathrm{S}$ & I & $\mathrm{S}$ & I & $\mathrm{R}$ & I & S & S & $\mathrm{R}$ & $\mathrm{S}$ & $\mathrm{S}$ & $\mathrm{R}$ & I & I & I & S & I \\
\hline P13 & DI & 37 & 5 & 22 & 7 & 69 & 6 & 8 & 8 & 0 & 0 & 2 & 17 & 0 & 0 & 9 & 32 & 6 & 0 & 0 & 0 & 13 & 0 \\
\hline & $\mathrm{R}$ & $\mathrm{S}$ & $\mathrm{R}$ & S & $\mathrm{R}$ & $S$ & $\mathrm{R}$ & $\mathrm{R}$ & $\mathrm{R}$ & I & I & $\mathrm{R}$ & S & I & I & $\mathrm{R}$ & S & $\mathrm{R}$ & I & I & I & $\mathrm{S}$ & I \\
\hline P14 & DI & 46 & 19 & 7 & 2 & 63 & 8 & 17 & 25 & 0 & 0 & 0 & 37 & 11 & 1 & 5 & 50 & 2 & 0 & 0 & 17 & 15 & 0 \\
\hline & $\mathrm{R}$ & $\mathrm{S}$ & $\mathrm{S}$ & $\mathrm{R}$ & $\mathrm{R}$ & $\mathrm{S}$ & $\mathrm{R}$ & $\mathrm{S}$ & $\mathrm{S}$ & I & I & I & $\mathrm{S}$ & $\mathrm{S}$ & $\mathrm{R}$ & $\mathrm{R}$ & $\mathrm{S}$ & $\mathrm{R}$ & I & I & $\mathrm{S}$ & $\mathrm{S}$ & I \\
\hline P15 & DI & 57 & 19 & 7 & 2 & 63 & 8 & 17 & 25 & 19 & 0 & 0 & 37 & 11 & 1 & 5 & 50 & 2 & 0 & 0 & 17 & 6 & 0 \\
\hline & $\mathrm{R}$ & S & $\mathrm{S}$ & $\mathrm{R}$ & $\mathrm{R}$ & S & $\mathrm{R}$ & S & $\mathrm{S}$ & $\mathrm{S}$ & I & I & S & $\mathrm{S}$ & $\mathrm{R}$ & $\mathrm{R}$ & $\mathrm{S}$ & $\mathrm{R}$ & I & I & $\mathrm{S}$ & $\mathrm{R}$ & I \\
\hline P16 & DI & 67 & 8 & 19 & 0 & 72 & 11 & 14 & 22 & 2 & 0 & 26 & 26 & 0 & 0 & 0 & 40 & 0 & 0 & 0 & 0 & 25 & 0 \\
\hline & $\mathrm{R}$ & S & $\mathrm{R}$ & S & I & $\mathrm{S}$ & $\mathrm{S}$ & S & $\mathrm{S}$ & $\mathrm{R}$ & I & $S$ & $\mathrm{~S}$ & I & I & I & $\mathrm{S}$ & I & I & I & I & $\mathrm{S}$ & I \\
\hline P17 & DI & 70 & 39 & 65 & 21 & 78 & 44 & 69 & 36 & 37 & 0 & 10 & 44 & 3 & 19 & 20 & 46 & 5 & 0 & 0 & 13 & 15 & 0 \\
\hline & $\mathrm{R}$ & $\mathrm{S}$ & S & $\mathrm{S}$ & $\mathrm{S}$ & $\mathrm{S}$ & $\mathrm{S}$ & $\mathrm{S}$ & S & S & I & S & $\mathrm{S}$ & $R$ & $\mathrm{~S}$ & $\mathrm{~S}$ & $\mathrm{~S}$ & $R$ & I & I & $\mathrm{S}$ & $\mathrm{S}$ & I \\
\hline P18 & DI & 44 & 7 & 63 & 5 & 12 & 12 & 4 & 0 & 0 & 1 & 7 & 20 & 2 & 2 & 0 & 45 & 0 & 0 & 0 & 7 & 16 & 0 \\
\hline & $\mathrm{R}$ & $\mathrm{S}$ & $\mathrm{R}$ & $\mathrm{S}$ & $\mathrm{R}$ & $\mathrm{S}$ & $\mathrm{S}$ & $\mathrm{R}$ & I & I & $\mathrm{R}$ & $\mathrm{R}$ & $\mathrm{S}$ & $\mathrm{R}$ & $\mathrm{R}$ & I & $\mathrm{S}$ & I & I & I & $\mathrm{R}$ & $\mathrm{S}$ & I \\
\hline P19 & DI & 65 & 22 & 13 & 4 & 54 & 18 & 1 & 4 & 0 & 0 & 0 & 26 & 3 & 0 & 6 & 20 & 1 & 0 & 0 & 3 & 0 & 0 \\
\hline & $\mathrm{R}$ & $S$ & $\mathrm{~S}$ & $S$ & $\mathrm{R}$ & $\mathrm{S}$ & $S$ & $\mathrm{R}$ & $\mathrm{R}$ & I & I & I & S & $\mathrm{R}$ & I & $\mathrm{R}$ & $\mathrm{S}$ & $\mathrm{R}$ & I & I & $\mathrm{R}$ & I & I \\
\hline P20 & DI & 67 & 8 & 19 & 11 & 72 & 0 & 8 & 22 & 2 & 0 & 26 & 26 & 11 & 0 & 0 & 40 & 0 & 0 & 0 & 0 & 25 & 0 \\
\hline & $\mathrm{R}$ & S & $\mathrm{R}$ & S & $\mathrm{S}$ & $\mathrm{S}$ & I & $\mathrm{R}$ & $S$ & $\mathrm{R}$ & I & $\mathrm{S}$ & $\mathrm{S}$ & $\mathrm{S}$ & I & I & S & I & I & I & I & $\mathrm{S}$ & I \\
\hline P21 & DI & 22 & 39 & 70 & 17 & 61 & 14 & 23 & 0 & 6 & 31 & 0 & 21 & 9 & 0 & 3 & 61 & 34 & 0 & 0 & 19 & 33 & 0 \\
\hline & $\mathrm{R}$ & $\mathrm{S}$ & $\mathrm{S}$ & $\mathrm{S}$ & $\mathrm{S}$ & $\mathrm{S}$ & $\mathrm{S}$ & $\mathrm{S}$ & I & $\mathrm{R}$ & S & I & $\mathrm{S}$ & $\mathrm{R}$ & I & $\mathrm{R}$ & $\mathrm{S}$ & S & I & I & $\mathrm{S}$ & S & I \\
\hline P22 & DI & 72 & 2 & 27 & 28 & 67 & 44 & 14 & 36 & 20 & 27 & 0 & 42 & 0 & 0 & 0 & 61 & 15 & 0 & 0 & 50 & 17 & 0 \\
\hline & $\mathrm{R}$ & $\mathrm{S}$ & $\mathrm{R}$ & $\mathrm{S}$ & $\mathrm{S}$ & $\mathrm{S}$ & S & $\mathrm{S}$ & $\mathrm{S}$ & $S$ & $\mathrm{~S}$ & I & $\mathrm{S}$ & I & I & I & $\mathrm{S}$ & S & I & I & $\mathrm{S}$ & $\mathrm{S}$ & I \\
\hline P23 & DI & 37 & 5 & 22 & 7 & 69 & 6 & 8 & 8 & 0 & 0 & 2 & 17 & 0 & 0 & 9 & 32 & 6 & 0 & 0 & 0 & 13 & 0 \\
\hline & $\mathrm{R}$ & $\mathrm{S}$ & $\mathrm{R}$ & $\mathrm{S}$ & $\mathrm{R}$ & $\mathrm{S}$ & $\mathrm{R}$ & $\mathrm{R}$ & $\mathrm{R}$ & $\mathrm{R}$ & I & $\mathrm{R}$ & $\mathrm{S}$ & I & I & $R$ & $\mathrm{~S}$ & $\mathrm{R}$ & I & I & I & $\mathrm{S}$ & I \\
\hline P24 & DI & 67 & 24 & 37 & 29 & 33 & 5 & 13 & 7 & 0 & 8 & 0 & 0 & 12 & 6 & 0 & 57 & 19 & 0 & 3 & 32 & 61 & 0 \\
\hline & $\mathrm{R}$ & $\mathrm{S}$ & $\mathrm{S}$ & $S$ & $\mathrm{~S}$ & S & $\mathrm{R}$ & S & $\mathrm{R}$ & I & $\mathrm{R}$ & I & I & $\mathrm{S}$ & $\mathrm{R}$ & I & $\mathrm{S}$ & $\mathrm{S}$ & I & $\mathrm{R}$ & $\mathrm{S}$ & $\mathrm{S}$ & I \\
\hline P25 & DI & 33 & 28 & 0 & 1 & 22 & 17 & 2 & 11 & 0 & 0 & 0 & 19 & 0 & 0 & 0 & 28 & 0 & 0 & 0 & 11 & 6 & 0 \\
\hline & $\mathrm{R}$ & $\mathrm{S}$ & $\mathrm{S}$ & I & $\mathrm{R}$ & $\mathrm{S}$ & $\mathrm{S}$ & $\mathrm{R}$ & S & I & I & I & $\mathrm{S}$ & I & I & I & $S$ & I & I & I & $\mathrm{S}$ & $\mathrm{R}$ & I \\
\hline P26 & DI & 65 & 11 & 14 & 15 & 52 & 9 & 32 & 0 & 0 & 21 & 3 & 2 & 0 & 14 & 0 & 48 & 19 & 0 & 0 & 11 & 32 & 0 \\
\hline & $\mathrm{R}$ & S & $\mathrm{S}$ & $\mathrm{S}$ & $\mathrm{S}$ & $\mathrm{S}$ & $R$ & $\mathrm{~S}$ & I & I & $\mathrm{S}$ & $\mathrm{R}$ & $\mathrm{R}$ & I & $S$ & I & $\mathrm{S}$ & S & I & I & $\mathrm{S}$ & S & I \\
\hline P27 & DI & 57 & 31 & 24 & 4 & 56 & 12 & 1 & 4 & 0 & 0 & 3 & 26 & 0 & 0 & 4 & 24 & 1 & 0 & 0 & 0 & 3 & 0 \\
\hline & $\mathrm{R}$ & $\mathrm{S}$ & $\mathrm{S}$ & $\mathrm{S}$ & $\mathrm{R}$ & $\mathrm{S}$ & $\mathrm{S}$ & $\mathrm{R}$ & $\mathrm{R}$ & I & I & $\mathrm{R}$ & $\mathrm{S}$ & I & I & $\mathrm{R}$ & $\mathrm{S}$ & $\mathrm{R}$ & I & I & I & $\mathrm{R}$ & I \\
\hline P28 & DI & 56 & 39 & 46 & 21 & 78 & 44 & 16 & 36 & 37 & 1 & 10 & 44 & 3 & 19 & 20 & 46 & 5 & 0 & 5 & 15 & 13 & 0 \\
\hline & $\mathrm{R}$ & $S$ & S & $\mathrm{S}$ & $\mathrm{S}$ & $\mathrm{S}$ & $\mathrm{S}$ & $\mathrm{S}$ & S & $\mathrm{S}$ & $\mathrm{R}$ & $\mathrm{S}$ & S & $\mathrm{R}$ & S & S & S & $\mathrm{R}$ & I & $\mathrm{R}$ & $\mathrm{S}$ & $\mathrm{S}$ & I \\
\hline P29 & DI & 63 & 3 & 20 & 17 & 37 & 21 & 17 & 16 & 39 & 32 & 3 & 15 & 5 & 15 & 10 & 59 & 23 & 0 & 1 & 36 & 36 & 0 \\
\hline & $\mathrm{R}$ & $\mathrm{S}$ & $\mathrm{R}$ & $\mathrm{S}$ & $\mathrm{S}$ & $\mathrm{S}$ & $\mathrm{S}$ & $\mathrm{S}$ & $\mathrm{S}$ & S & $\mathrm{S}$ & $\mathrm{R}$ & $\mathrm{S}$ & $\mathrm{R}$ & S & $S$ & S & $\mathrm{S}$ & I & $\mathrm{R}$ & $\mathrm{S}$ & $\mathrm{S}$ & I \\
\hline P30 & DI & 52 & 6 & 42 & 5 & 12 & 12 & 16 & 0 & 0 & 1 & 7 & 20 & 2 & 2 & 0 & 45 & 0 & 0 & 0 & 16 & 17 & 0 \\
\hline & $\mathrm{R}$ & $\mathrm{S}$ & $\mathrm{R}$ & $\mathrm{S}$ & $\mathrm{R}$ & $\mathrm{S}$ & $\mathrm{S}$ & $\mathrm{S}$ & I & I & $\mathrm{R}$ & $\mathrm{R}$ & S & $\mathrm{R}$ & $\mathrm{R}$ & I & $\mathrm{S}$ & I & I & I & $\mathrm{S}$ & $\mathrm{S}$ & I \\
\hline P31 & DI & 46 & 16 & 34 & 5 & 58 & 14 & 8 & 7 & 0 & 0 & 7 & 29 & 0 & 0 & 6 & 44 & 3 & 0 & 0 & 0 & 16 & 0 \\
\hline & $\mathrm{R}$ & $\mathrm{S}$ & S & $\mathrm{S}$ & $\mathrm{R}$ & S & $\mathrm{S}$ & $\mathrm{R}$ & $\mathrm{R}$ & I & I & $\mathrm{R}$ & $\mathrm{S}$ & I & I & $\mathrm{R}$ & $\mathrm{S}$ & $\mathrm{R}$ & I & I & I & $\mathrm{S}$ & I \\
\hline P32 & DI & 73 & 16 & 63 & 2 & 62 & 24 & 9 & 7 & 0 & 0 & 0 & 23 & 12 & 1 & 34 & 58 & 5 & 0 & 0 & 7 & 9 & 0 \\
\hline & $\mathrm{R}$ & $\mathrm{S}$ & $\mathrm{S}$ & $\mathrm{S}$ & $\mathrm{R}$ & $\mathrm{s}$ & $\mathrm{S}$ & $R$ & $\mathrm{R}$ & I & I & I & $\mathrm{S}$ & $\mathrm{S}$ & $\mathrm{R}$ & S & $\mathrm{S}$ & $\mathrm{R}$ & I & I & $\mathrm{R}$ & $R$ & I \\
\hline P33 & DI & 35 & 22 & 8 & 3 & 18 & 13 & 5 & 14 & 2 & 0 & 3 & 25 & 0 & 0 & 0 & 36 & 0 & 0 & 0 & 15 & 4 & 0 \\
\hline & $\mathrm{R}$ & $\mathrm{S}$ & $\mathrm{S}$ & $\mathrm{R}$ & $\mathrm{R}$ & $\mathrm{S}$ & $\mathrm{S}$ & $\mathrm{R}$ & $S$ & $\mathrm{R}$ & I & $\mathrm{R}$ & $\mathrm{S}$ & I & I & I & $\mathrm{S}$ & I & I & I & $\mathrm{S}$ & $\mathrm{R}$ & I \\
\hline P34 & DI & 38 & 26 & 4 & 7 & 24 & 19 & 3 & 12 & 1 & 0 & 5 & 17 & 0 & 0 & 0 & 27 & 0 & 0 & 0 & 12 & 9 & 0 \\
\hline & $\mathrm{R}$ & $\mathrm{S}$ & $\mathrm{S}$ & $\mathrm{R}$ & $\mathrm{R}$ & $\mathrm{S}$ & $\mathrm{S}$ & $\mathrm{R}$ & $\mathrm{S}$ & $\mathrm{R}$ & I & $\mathrm{R}$ & $\mathrm{S}$ & I & I & I & $\mathrm{S}$ & I & I & I & $\mathrm{S}$ & $\mathrm{R}$ & I \\
\hline
\end{tabular}

Note: Disease index $<10$ meaned Resistant (R), Disease index $\geq 10$ meaned susceptible (S), Disease index $=0$ meaned immune (I). From CR1 to CR21: Chunqiushenggen, CR65, Tianci, Jinfu baby cabbage, Guizu, Degaorongyao, Kanggen 911, Taineng CR119, Beisheng CR12, Chinese cabbage King, CR Mogen, Xinkanggen, CR Gaokangwang, Kangbingwang CR117, Guoshen CR167, Shenggen No.1, Shangpin, Shangpin CR527, Degao CR117, Tiejia No.1, Shangpin CR523

Pathotypes of P. brassicae and their distribution in Yunnan are shown in Fig. 2.

Clubroot disease was detrimental in Kunming and its surrounding areas, Fumin, Anning, Jinning, Songming and Yiliang. Kunming became a disease centre, spreading outward to some areas of Chuxiong, Dali, Dehong, Lijiang, 
Han et al. / Intl J Agric Biol, Vol 25, No 4, 2021

Table 5: Identification results of molecular markers

\begin{tabular}{|c|c|c|c|c|c|c|c|c|}
\hline \multirow[t]{2}{*}{ Code } & \multirow[t]{2}{*}{ Cultivars } & \multicolumn{7}{|c|}{ Resistant site } \\
\hline & & $\mathrm{Crrl}$ & $\mathrm{Crr} 2$ & $\mathrm{Crr} 3$ & $C R a$ & $C R b$ & $C R c$ & $C R k$ \\
\hline 1 & BS & 1 & - & 1 & - & - & 1 & + \\
\hline 2 & JQ & 1 & - & 1 & - & - & 1 & + \\
\hline 3 & WB & 1 & - & - & \pm & + & - & + \\
\hline 4 & LT & - & - & + & \pm & + & + & + \\
\hline 5 & 83-1 (CK) & - & 1 & - & - & - & - & + \\
\hline 6 & CR1 & - & - & - & - & - & - & \pm \\
\hline 7 & CR2 & - & - & - & - & - & - & \pm \\
\hline 8 & CR3 & 1 & - & - & - & - & - & \pm \\
\hline 9 & CR4 & - & 1 & - & - & - & - & \pm \\
\hline 10 & CR5 & - & + & - & - & - & - & \pm \\
\hline 11 & CR6 & - & - & - & - & - & - & \pm \\
\hline 12 & CR7 & - & - & - & - & - & - & - \\
\hline 13 & CR8 & - & - & - & - & - & - & \pm \\
\hline 14 & CR9 & 1 & - & - & - & - & - & - \\
\hline 15 & CR10 & - & - & - & - & - & - & \pm \\
\hline 16 & CR 11 & - & - & - & - & - & - & - \\
\hline 17 & CR12 & 1 & - & - & - & - & - & \pm \\
\hline 18 & CR13 & - & - & - & - & - & - & \pm \\
\hline 19 & CR14 & - & - & - & - & - & - & \pm \\
\hline 20 & CR15 & - & - & - & \pm & - & - & \pm \\
\hline 21 & CR16 & 1 & - & - & - & - & - & \pm \\
\hline 22 & CR17 & - & - & - & + & + & - & \pm \\
\hline 23 & CR18 & - & - & - & + & + & + & \pm \\
\hline 24 & CR19 & - & - & - & - & - & - & \pm \\
\hline 25 & CR20 & - & - & - & - & - & - & \pm \\
\hline 26 & CR21 & - & - & - & + & + & + & \pm \\
\hline
\end{tabular}

Table 6: Resistance varieties of different Pathotypes

\begin{tabular}{ll}
\hline Race & Cultivars \\
\hline Race 1 & Taineng CR119, Beisheng CR12, Chinese cabbage King, Shangpin, Shangpin CR527, Degao CR117, ShangpinCR523 \\
Race 2 & Shangpin, Shangpin CR527, Shangpin CR523 \\
Race 4 & Shangpin \\
Race 10 & Beisheng CR12, Shangpin, Shangpin CR527, Shangpin CR523 \\
Race 14 & Beisheng CR12, CR Gaokangwang, Kangbingwang CR117, Shenggen NO.1, Shangpin, Shangpin CR527, Degao CR117, Shangpin CR523 \\
\hline
\end{tabular}

Honghe and Qujing, and to counter this expansion, we propose prevention and control strategies for clubroot disease in Yunnan Province. Although planting diseaseresistant Chinese cabbage varieties or non-cruciferous crops in clubroot disease areas can reduce the number of $P$. brassicae spores in the soil and avoid the occurrence of clubroot, we need to invoke quarantine and disinfection of the seeds and seedlings in the adjacent areas of the disease area, avoiding soil pollution caused by $P$. brassicae from clubroot disease areas and preventing the expansion of the disease.

The pathogenicity of $P$. brassicae in Yunnan Province has changed over the past decade. The $P$. brassicae found in Lufeng was pathotype 12 in 2013 (Liu et al. 2013), but is now pathotype 2 . Both of the 2 P. brassicae samples from Songming were pathotype 4 in our study, but pathotype 7 was identified in 2016 (Chen et al. 2016). Pathotype 2 had higher pathogenicity than 12 , and pathotype 4 was higher than pathotype 7 . These results indicate that clubroot disease in Lufeng and Songming had become more robust over time. Pathogenicity of pathotype 4 was the highest among all pathotypes. However, only 3 cultivars: Shangpin, Shangpin CR523 and Shangpin CR527, were resistant to all types of pathotype 4 in Yunnan; the other varieties expressed either immune, resistant or susceptible responses depending on the type of pathogen 4 . The responses of the same cultivar to 24 samples of pathotype 4 were different, which indicated its pathogenicity differences and complexity. It was reported by Li et al. (2012) that they had successfully isolated several pathotypes (2, 4, 8 and 11) from pathotype 4 samples in Shenyang Province by separating single resting spores. Their results led to the assumption that pathotype 4 samples in Yunnan may be composed of different types of $P$. brassicae. At present, these types cannot be clearly distinguished using the Williams system. Therefore, a more accurate identification method is crucial for the prevention and control of clubroot, and for clubroot-resistant breeding.

The application of resistant varieties is the most economical, effective and sustainable measure for the control of clubroot. In this study, Shangpin, Shangpin CR527 and Shangpin CR523 were immune to pathotypes 1, 
2, 10 and 14 of $P$. brassicae, however, only Shangpin was immune to all of the types of pathotype 4 of $P$. brassicae (Table 6). These results provide a reference for the selection of Chinese cabbage cultivars in the clubroot disease area of Yunnan Province. They could also provide germplasm for the breeding of clubroot-resistant Chinese cabbage.

Some molecular markers of clubroot resistance have been identified. $C R a$ and $C R b$ are alleles or closely linked disease resistance loci on the A03 chromosome (Kato et al. 2012; Hatakeyama et al. 2017). Chinese cabbage with $C R a$ and $C R b$ could be resistant to pathotype 4 of $P$. brassicae (Saravanan et al. 2003) $C R a$ and $C R b$ loci were detected in Shangpin, Shangpin CR527 and Shangpin CR523 in this study. Shangpin, was only detected in three resistant markers. These three markers were also detected in Shangpin CR523 and Shangpin CR527, but their resistance was lower, indicating that there are still undiscovered resistance sites in Shangpin, and need further research. According to Sakamoto et al. (2008), Crr3 and CRk are two independent resistance loci on chromosome A03, with a genetic distance of $7 \mathrm{cM}$. However, in this experiment, the two resistance loci were quite different. The $C r r 3$ locus was not detected in the 21 tested varieties, but $C R k$ loci were widely detected, even in the susceptible control ' $83-1$ '. It is suspected that $C R k$ loci may not be closely related to disease resistance genes.

Beisheng CR12 (CR9) was immune to P1-P4, P12$\mathrm{P} 17, \mathrm{P} 22, \mathrm{P} 23, \mathrm{P} 25, \mathrm{P} 27$ and $\mathrm{P} 31-\mathrm{P} 34$, relating to pathotypes $1,4,10$ and 14 (Table 3 ), but did not carry any known resistance genes (Table 5), indicating that unknown resistant genes were present. Beisheng CR12 would be a potential genetic resource for developing resistant markers or genes to prevent clubroot.

\section{Conclusion}

P. brassicae was more complex and pathogenic in Yunnan than in other areas of China. The two different strategies and three cultivars were suggested to prevent and control clubroot. Further studies are needed to find the potential genes resistant-clubroot in cultivars Beisheng CR12 and Shangpin.

\section{Acknowledgements}

This work was supported financially by the Key Research and Development Project of Yunnan Province (No. 2018BB021). We are thankful to the Vegetable Industry Technology System Experimental Stations in Jianshui, Dehong, Tonghai, Luliang, Kunming and Ludian for collection of $P$. brassicae root samples.

\section{Author Contributions}

Designed the experiments: YLY. Performed the experiments: JLY, XYH and EZL.Analyzed the data: XYH and JLY. Contributed reagents/materials/analysis tools: IU and EZL. Wrote the paper: XYH and YLY.

\section{Conflict of Interest}

We, the authors, declare no conflict of interest of any kind among ourselves of the institutions where the work was done

\section{Data Availability Declaration}

All data reported in this article are available with the corresponding author and will be produced on demand

\section{References}

Chen J, XS Ren, HY Song, CQ Li, TC Yuan, J Si (2016). Identification of races of Plasmodiophora brassicae from four different regions and resistance identification of new cabbage cross combinations. $J$ Southwest Univ 38:67-72

Faggian R, SR Bulman, AC Lawrie, IJ Porter (1999). Specific polymerase chain reaction primers for the detection of Plasmodiophora brassicae in soil and water. Phytopathology 89:392-397

Fei WX, SF Wang, BC Hu, QS Li, SM Hou, SB Rong, XY Wu (2015). Control effect of different fungicides on clubroot and yield loss caused by Plasmodiophorabrassicae in Brassicanapus.PlantProt 41:201-204

Hatakeyama K, T Niwa, T Kato, T Ohara, T Kakizaki, S Matsumoto (2017). The tandem repeated organization of NB-LRR genes in the clubroot-resistant $C R b$ locus in Brassica rapa L. Mol Genet Genomics 292:397-405

Hatakeyama K, K Suwabe, RN Tomita, T Kato, T Nunome, H Fukuoka, S Matsumoto (2013). Identification and characterization of Crrla, a gene for resistance to clubroot disease (Plasmodiophora brassicae Woronin) in Brassica rapa L. PLoS One 8; Article e54745

Hirai M, T Harada, N Kubo, M Tsukada, K Suwabe, S Matsumoto (2004). A novel locus for clubroot resistance in brassica rapa and its linkage markers. Theor Appl Genet 108:639-643

Howard RJ, SE Strelkov, MW Harding (2010). Clubroot of cruciferous crops - new perspectives on an old disease. Can J Plant Pathol 32:43-57

Karling JS (1942). The Plasmodiophorales, $2^{\text {nd }}$ edn. Springer, New York, USA

Kato T, K Hatakeyama, N Fukino, S Matsumoto (2012). Identificaiton of a clubroot resistance locus conferring resistance to a Plasmodiophora brassicae classified into pathotype group 3 in Chinese cabbage (Brassica rapa $\mathrm{L}$ ). Breed Sci 62:282-287

Lee JE, JS Kang, YW Ki, JH Park, IC Shin, HC Koh (2012). Fluazinam targets mitochondrial complex I to induce reactive oxygen speciesdependent cytotoxicity in SH-SY5Y cells. Neurochem Intl 60:773-781

Lee SO, GJ Choi, YH Choi, KS Jang, JC Kim (2008). Isolation and characterization of endophytic actinomycetes from Chinese cabbage roots as antagonists to Plasmodiophora brassicae. Microbiol Biotechnol 18:1741-1746

Li Q, XQ Shen, XC Geng, L Li (2012). Separate inoculation of single resting spores and identification of Plasmodiophora brassicae races. Plant Prot 38:95-101

Liu F, LH Zhang, GH Ji (2013). Identification of physiological race of Plasmodiophora brassicae in Yunnan and Tibet. Chin Veg 20:77-81

Lv B, Y Wang, H Xai, Z Yao, C Jiang (2018). Differences in effects of different amendments on growth and soil fertility of Chinese cabbage in yellow brown soil and red soil. Sci Agric Sin 51:4306-4315

Mcgrann GRD, T Yoxall, LJ Paterson, JMG Taylor, IG Birmpilis, DR Walters, ND Havis (2017). Control of light leaf spot and clubroot in brassica crops using defence elicitors. Eur J Plant Pathol 148:447-461

Peng G, D Pageau, SE Strelkov, BD Gossen, S Hwang, R Lahlali (2015). A >2-year crop rotation reduces resting spores of Plasmodiophora brassicae in soil and the impact of clubroot on canola. Eur J Agron $70: 78-84$ 
Peng SS, ZH Ren, XL Huang, MJ Liu, LF Sun, EM Liu (2013). Physiological race identification on clubroot of cruciferous crops caused by Plasmodiophora brassicae in Hunan. J Changj Veg 6:46-49

Sakamoto K, A Saito, N Hayashida, G Taguchi, E Matsumoto (2008). Mapping of isolate-specific QTLs for clubroot resistance in Chinese cabbage (Brassica rapa L. sspp. pekinensis). Theor Appl Genet 117:759-767

Saravanan T, M Muthusamy, T Marimuthu (2003). Development of integrated approach to manage the fusarial wilt of banana. Crop Prot 22:1117-1123

Shen XQ, K Nie, Q Wu, YG Zhang, XH Meng (2009). Initial research report on differentiation identification of Chinese cabbage clubroot main physiological races. Chin Veg 1:59-62

Suo H, LZ Chen, H Xu, B Song, XX Fan, XH Yuan, CZ He (2015). Research progress in clubroot of crucifers. J Anhui Agric Sci 126:115-117

Suwabe K, H Tsukazaki, H Iketani, K Hatakeyama, M Fujimura, T Nunome, H Fukuoka, S Matsumoto, M Hirai (2003). Identification of two loci for resistance to clubroot (Plasmodiophora brassicae woronin) in Brassica rapa L. Theor Appl Genet 107:997-1002

Tan C, YL Yue (2013). Identification of different Chinese cabbage [Brassica campestris L. sspp. pekinensis (Lour) Olsson] varieties resistance to clubroot (Plasmodiophora brassicae). Chin Veg 8:91-94
Ueno H, E Matsumoto, D Aruga, S Kitagawa, H Matsumura, N Hayashida (2012). Molecular characterization of the CRa gene conferring clubroot resistance in Brassica rapa. Plant Mol Biol 80:621-629

Wang SM, L Yin, T Shen, H Yang, B Shen (2016). Biological characteristics of Streptomyces albospinus CT205 and its biocontrol potential against cucumber fusarium wilt. Biocont Sci Technol 26:951-963

Webster MA, GR Dixon (1991). Calcium, pH and inoculum concentration influencing colonization by Plasmodiophora brassicae. Mycol Res 95:64-73

Williams PH (1966). A system for the determination of races of Plasmodiophora brassicae that infect cabbage and rutabaga. Phytopathology 56:624-626

Yin JL, ZY Qian, YF Zheng, CX Zhao, YL Yue (2018). Physiological race identification of Plasmodiophora brassicae and screening of resistant source in Chinese cabbage at Yingjiang County. Chin Veg 8:55-57

Yuan Q, IS Druzhinina, XY Pan, ZL Yuan (2016). Microbially mediated plant salt tolerance and microbiome-based solutions for saline agriculture. Biotechnol Adv 34:1245-1259

Zheng J, XL Wang, Xiao Y, SQ Wei, D Wang, Y Huang, WM Wang, H Yang (2019). Specificgenes identified in pathotype 4 of the clubroot pathogen Plasmodiophora brassicae. Plant Dis 103:495-503 